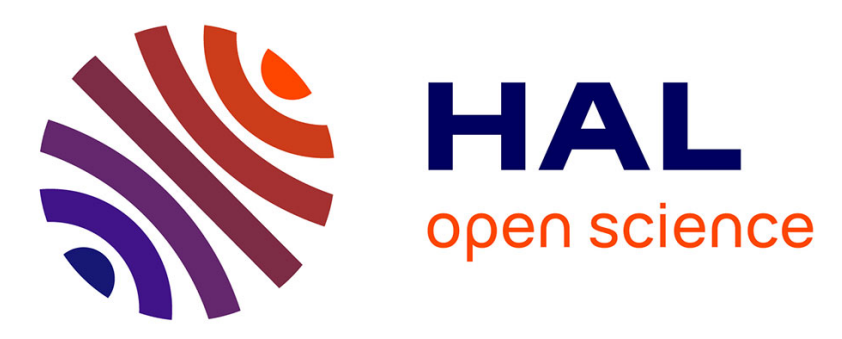

\title{
Chiral platinum-polypyrrole hybrid films as efficient enantioselective actuators
}

Sunpet Assavapanumat, Bhavana Gupta, Gerardo Salinas, Bertrand Goudeau, Chularat Wattanakit, Alexander Kuhn

\section{- To cite this version:}

Sunpet Assavapanumat, Bhavana Gupta, Gerardo Salinas, Bertrand Goudeau, Chularat Wattanakit, et al.. Chiral platinum-polypyrrole hybrid films as efficient enantioselective actuators. Chemical Communications, 2019, 55 (73), pp.10956-10959. 10.1039/C9CC05854K . hal-02509635

\section{HAL Id: hal-02509635 \\ https://hal.science/hal-02509635}

Submitted on 17 Mar 2020

HAL is a multi-disciplinary open access archive for the deposit and dissemination of scientific research documents, whether they are published or not. The documents may come from teaching and research institutions in France or abroad, or from public or private research centers.
L'archive ouverte pluridisciplinaire HAL, est destinée au dépôt et à la diffusion de documents scientifiques de niveau recherche, publiés ou non, émanant des établissements d'enseignement et de recherche français ou étrangers, des laboratoires publics ou privés. 
Chiral platinum-polypyrrole hybrid films as efficient enantioselective actuators

Sunpet Assavapanumat, ${ }^{\mathrm{a}, \mathrm{b}}$ BhavanaGupta, ${ }^{\mathrm{a}}$ Gerardo Salinas, ${ }^{\mathrm{a}}$ Bertrand Goudeau, ${ }^{\mathrm{a}}$ Chularat Wattanakit $^{\mathrm{b}^{*}}$ and Alexander Kuhn ${ }^{\mathrm{a}^{*}}$

We report the synthesis of a hybrid bilayer, being composed of a free-standing conducting polymer film and a layer of mesoporous metal, encoded with chiral features. The resulting structure constitutes an enantioselective actuator, which can be electrochemically addressed in a wireless way. The controlled discriminatory deformation of the film allows an easy readout of chiral information.

Nowadays the development of materials with sophisticated properties is of crucial importance as it allows increasing their performance and application potential. Hybrid materials, composed of at least two chemically different constituents, have been studied for several decades, aiming at the integration of multiple component properties in a synergistic way.1 One important field of application for such hybrid structures is the development of efficient actuation systems. A particularly interesting scientific challenge is to achieve wireless actuation as a function of different chemical stimuli, for applications ranging from controlled robotic motion to remote sensor manipulation. 2 In this context, biomimetic actuation is of special interest aiming at, among others, reproducing the behavior of muscles. It has been recently demonstrated that muscle tissue can interact differently with the two enantiomers of a drug molecule3 and it is therefore interesting to design an artificial analog, showing such an enantioselective actuation behavior. Thus, the first major component of the proposed hybrid structure is a material which enables selective molecular recognition. Molecular imprinting is an efficient strategy for the generation of specific recognition sites that can interact with target molecules in a highly selective and sensitive way.4 Usually, imprinting is carried out with polymeric matrices which can be used for molecular recognition after the removal of a given template.4,5 Such molecular imprinted polymers (MIP) emerged as interesting materials for potential applications ranging from biosensing and chromatography to nanofiltration and electrodialysis.4a, 6 In the specific case of enantioselective sensing, designer surfaces need to be engineered in such a way that they retain chiral features.7 Since many pharmaceutical compounds are chiral with the desired effect for only one of the two enantiomers, their selective recognition as well as separation is an important challenge. $8 \mathrm{MIPs}$ have been widely employed to generate chiral recognition sites in the presence of a chiral template, however, sometimes suffer from difficult template removal, poor mass transfer, low binding constants and slow binding kinetics.4a, 7b, 9 Substituting polymeric materials with metals is an interesting alternative option, but the recognition efficiency is limited by the active area in the case of flat surfaces.10 To overcome the problem of small active surface areas, mesoporous metals can be used instead as a matrix. They are well-known as highly efficient materials for various applications ranging from catalysis and drug delivery to chemical detection.11 Based on these interesting properties, their molecular imprinting with chiral features has been recently proposed.12 The designed analogous porous materials are stable after removal of the template, and retain their chiral character even for long periods of time. The high surface area not only increases significantly the number of recognition sites, but also improves the accessibility by the target molecules.

The second major ingredient of the here proposed hybrid material is a conducting polymer. Conducting polymers are well-known for showing electrochromic features and controlled actuation behavior, triggered by a change in oxidation state.13 Usually, in order to observe such effects, the polymer needs to be physically connected to a power supply. A rather recent and alternative option is to address conducting polymers in a wireless way, based on the concept of bipolar electrochemistry.14 When a free-standing polypyrrole (PPy) film is integrated in a bipolar electrochemical set-up, it undergoes reversible mechanical deformation, $2 \mathrm{c}$ which can be used for 
controlled actuation,15and also for wireless sensing of various types of molecules.16 In the latter case, the degree of bending of the PPy actuator at one extremity is directly correlated with the concentration of analyte that is converted at the opposite extremity. So far the selectivity of such an actuator has been rather limited and mostly controlled by the thermodynamic values of the involved redox couples.

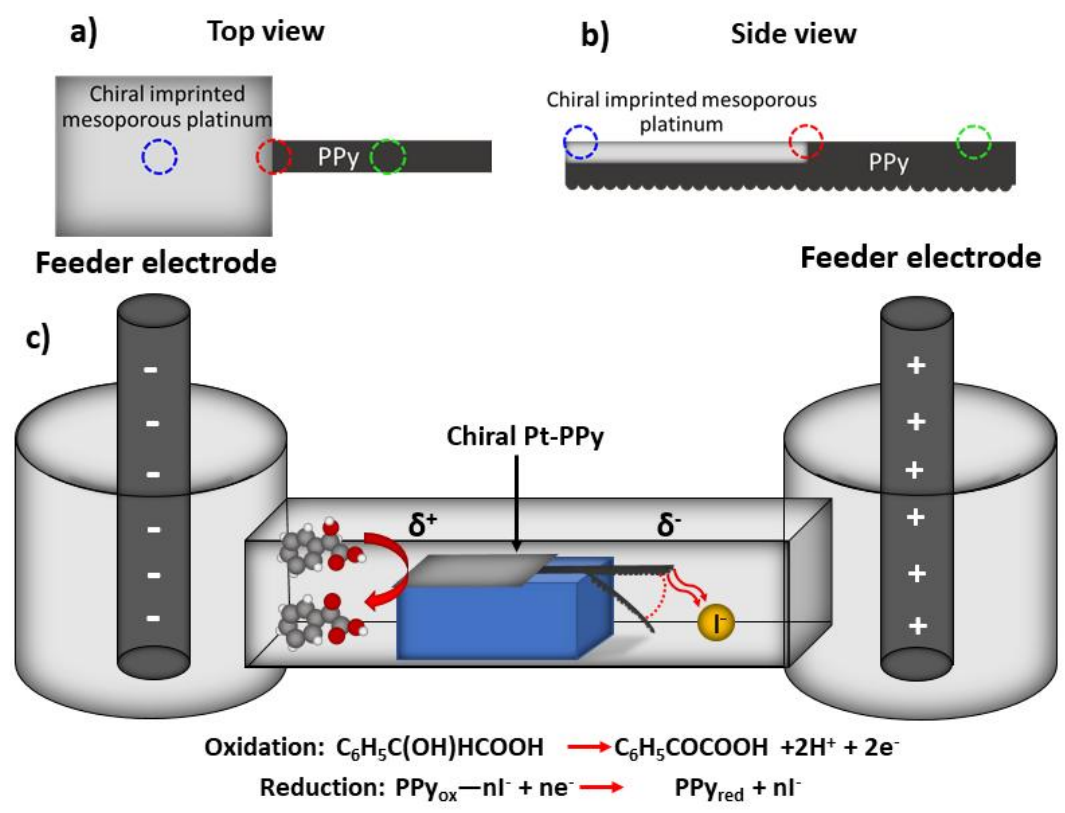

Scheme 1. Illustration of the hybrid Pt-PPy film used for chiral recognition (a) Top view and (b) cross section of Pt-PPy (c) Scheme of the bipolar electrochemical cell for chiral recognition of mandelic acid with the hybrid actuator.

In order to introduce a much higher degree of selectivity, we propose in the present contribution to integrate the above-mentioned chiral platinum as an enantioselective component into a wireless PPy actuator. The resulting hybrid material with double functionality allows triggering the actuation in the presence of a chosen enantiomer. This can be considered as the artificial biomimetic equivalent of a muscle which is preferentially interacting with only one stereoisomer, analog to what has been observed in body tissue. 3

The efficient wireless electromechanical deformation of PPy observed so far makes it a promising candidate for the integration of a chiral encoded mesoporous metal in order to obtain a freestanding hybrid material with enhanced functionalities. Such a bilayer structure can be readily addressed by bipolar electrochemistry, and exhibits enantioselective recognition properties, which directly influence the speed of deformation of the polymer. In order to intimately combine both materials and facilitate their synergistic action, successive electrodeposition steps have to be employed.

In a first step, chiral encoded mesoporous Pt was electrodeposited on a fraction of a gold coated glass slide (for details see Supporting Information and Fig. S1). Subsequently the substrate comprising the gold and the platinum layer is covered with a layer of PPy by electropolymerization. Then the gold layer is etched away with a $\mathrm{KI} / \mathrm{I} 2$ solution in order to liberate the hybrid material. Scheme $1 \mathrm{a}$ and $1 \mathrm{~b}$ represent the top and side view of the resulting free-standing film. Its different components, PPy (green spot), mesoporous Pt (blue spot), and the Pt-PPy junction (red spot)) have been characterized by Scanning Electron Microscopy (SEM) as illustrated in Fig. 1. A homogeneous 
thin platinum layer (1?m) is intimately connected to the thicker PPy film (50?m) (Fig. 1b and 1e). The elemental composition is confirmed by EDS mapping (inset of Figure 1b). The PPy part of the hybrid material exhibits two different faces, similar to what has been described previously.2c Very smooth PPy is observed for the top face (Fig. 1C), because it has been facing the gold substrate during polymerization (Fig. 1a). However, the face which during the growth was oriented towards the solution is rather rough (Fig. S2). The so-obtained composite film shows good mechanical stability and can be used for the subsequent selectivity studies. Mandelic acid (MA) enantiomers have been chosen in order to examine the chiral selectivity of the hybrid Pt-PPy material. In a preliminary experiment, the as-prepared chiral imprinted Pt-PPy film was first studied by differential pulse voltammetry (DPV) with the different enantiomer solutions in the oxidation region of mandelic acid ( 0.2 to $0.8 \mathrm{~V}$ vs $\mathrm{Ag} / \mathrm{AgCl}$ ). Fig. S3 illustrates the enantioselective features of the freestanding hybrid material. The signal amplitude of Pt-PPy imprinted with R-MA is significantly higher in a R-MA solution compared to the analog experiment in S-MA.
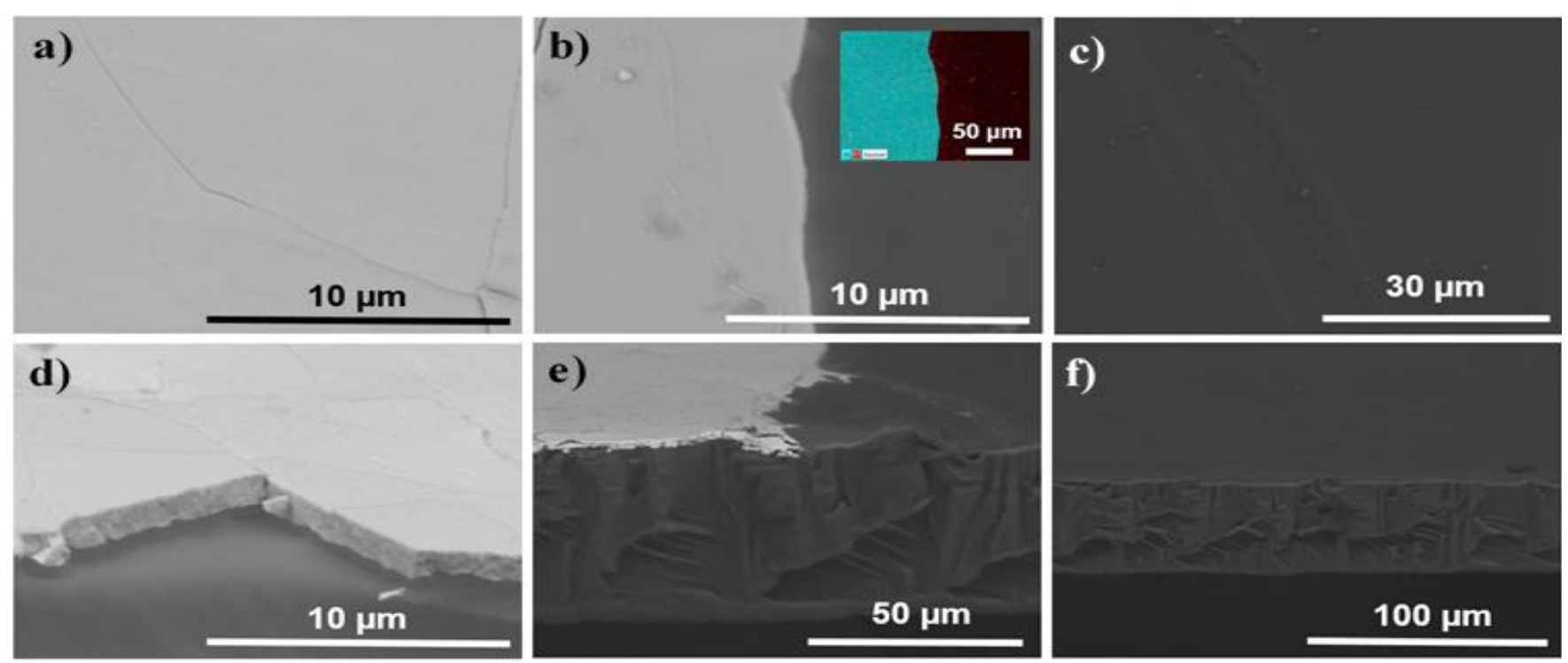

Fig. 1 SEM images of the hybrid chiral imprinted Pt-PPy film at different positions. (a,d) top and side view of the chiral-encoded platinum layer, $(b, e)$ top and side view of the Pt-PPy junction (inset: EDS map), (c,f) top and side view of the PPy film.

This indicates that the hybrid R-MA imprinted Pt-PPy preferentially converts R-MA at the platinum surface rather than S-MA. A very similar behavior has been observed in previous studies for the enantioselective electrochemistry of other chiral molecules.12a-12d, 17 As described in more detail in these previous reports and in the supporting information, the discrimination can be ascribed to a change in partition coefficient between the solution and the porous metal phase. One can compare the metal phase with a sponge that has a higher affinity for one enantiomer due to the presence of the chiral cavities. Therefore, the apparent concentration of the "good" enantiomer in the metal phase is higher with respect to the "wrong" enantiomer. This leads to higher oxidation currents in differential pulse voltammetry and it is therefore expected that in the bipolar electrochemical set-up such higher currents allow a similar discrimination via an electromechanical read-out.

For the bipolar electrochemical study, the free-standing chiral hybrid film was positioned in the middle of the cell with the rough surface oriented downwards (Scheme 1c). Applying the electric field 
with the chiral-encoded Pt facing, the negative feeder electrode leads to a positive polarization of this extremity $(\delta+)$. Above a certain threshold polarization, this allows mandelic acid to be oxidized at the chiral imprinted metal surface.18 On the other hand, the opposite PPy extremity acquires a negative polarization ( $\delta-)$, thus undergoes a reduction of the oxidized PPy, accompanied by a release of iodide anions in order to ensure charge compensation. lodide has been previously integrated into the polymer by ion exchange during the rather long ( $48 \mathrm{hrs}$ ) gold etching step. The release of iodide during polarization preferentially occurs through the rough face of the polymer due to the higher active surface area. Consequently, this face of the polymer strip starts to shrink and induces a bending towards the rough face. The degree of bending should be therefore directly correlated with the amount of electrons available from the oxidation of mandelic acid occurring at the opposite end of the strip.

In order to verify this hypothesis, the hybrid film is exposed to the electric field in the presence of the two different enantiomers and the bending is measured as the distance between the PPy extremity before and during the application of the electric field. The chiral selectivity can then be deduced from the difference in relative bending:

Relative bending $=($ Bending distance for the studied enantiomer at a given time $) /($ Final bending distance for the imprinted enantiomer) (1)

R-MA and S-MA imprinted Pt-PPy samples were used as actuators with an enantioselectivity illustrated in Figure 2. In the case of R-MA imprinted Pt-PPy, the polymer strip exhibits a significant and rapid bending in R-MA solution due to the more efficient electrooxidation of this enantiomer. However, in a solution containing S-MA, the target molecules are converted more slowly and therefore lead to a less pronounced bending (Fig. 2a and Video S1). In order to verify the concept, the symmetrically opposite situation is also studied by using S-MA imprinted Pt-PPy. As expected, the bending is now more efficient in S-MA solution compared to the R-MA solution (Fig. $2 \mathrm{~b}$ and Video S2). A final control experiment with non-imprinted

Pt-PPy leads to no significant difference between the two enantiomer solutions, due to the achiral character of the actuator (Fig. S4). 

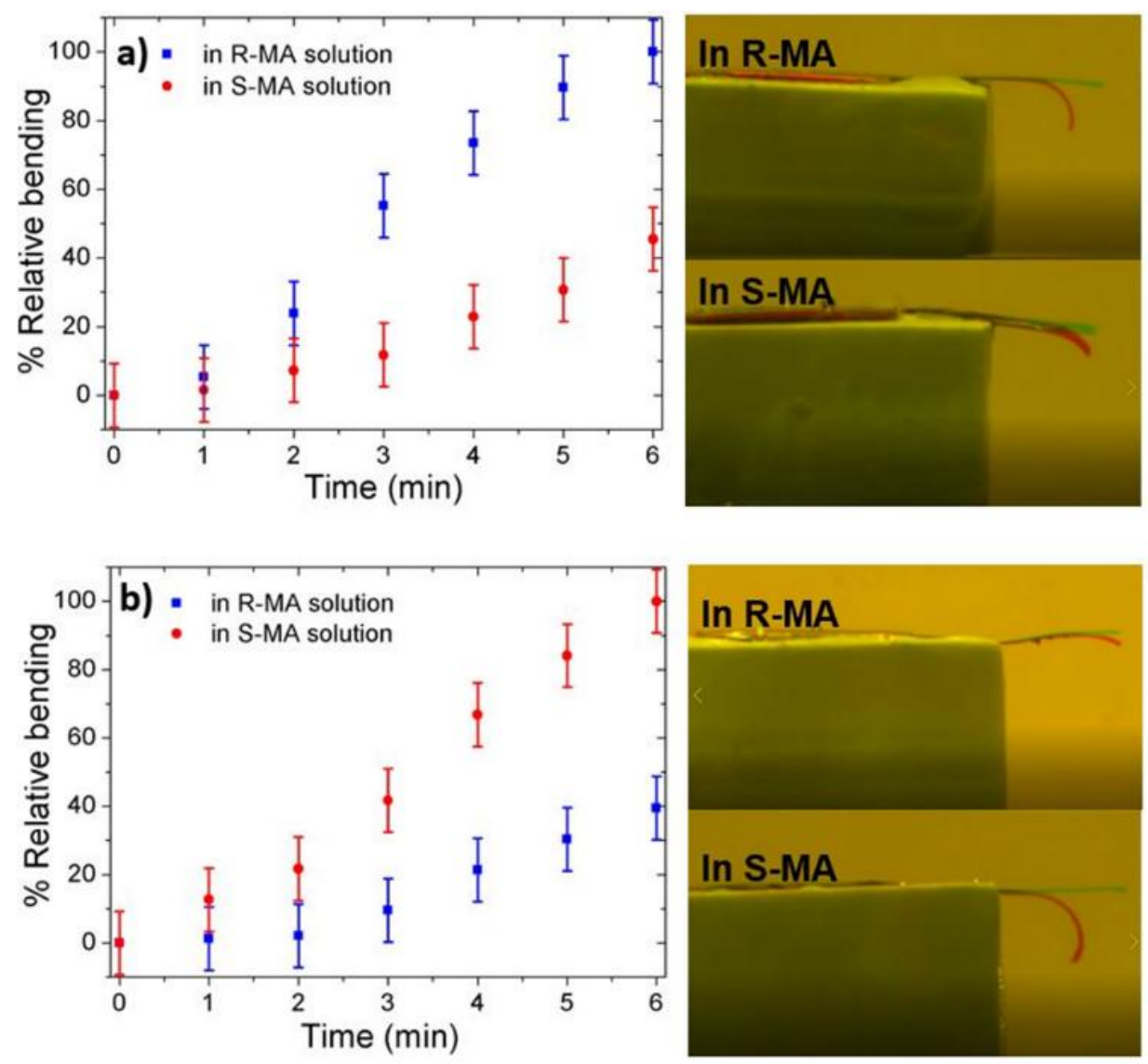

Fig. 2 Bipolar electrochemical actuation of hybrid chiral Pt-PPy in 50 mM R-MA (blue) and S-MA (red) using $50 \mathrm{mM} \mathrm{HCl}$ as supporting electrolyte. Relative bending measured as a function of time for (a) RMA imprinted Pt-PPy and (b) S-MA imprinted Pt-PPy. The pictures on the right have been taken after $6 \mathrm{~min}$ of applied potential. The green line indicates the initial position of the actuator, whereas red corresponds to its final position.

The differentiation between the two enantiomers is by essence based on the kinetics of bending, because it is directly related to the amplitude of the local current flowing in the bipolar object. The analytical information is provided by the speed of bending of these materials conditioned by the selective recognition via the chiral imprinted mesoporous platinum. The above results indicate that the bending rate is significantly different for the two enantiomers and thus allows achieving a pronounced enantioselectivity of these hybrid materials. Therefore, at a given time, the degree of bending is higher for the "good" enantiomer compared to the "wrong" one. Obviously, when waiting for a long enough time this difference will become smaller, because the polymer in the "good" enantiomer solution reaches its maximum degree of bending imposed by the experimental set-up, and therefore the polymer in the "wrong" solution will be able to catch up.

In summary, an original hybrid bilayer material, composed of chiral imprinted platinum and polypyrrole, has been successfully synthesized by electrodeposition and electropolymerization. The unique features of both compounds, chiral recognition and wireless actuation abilities, are combined in a synergistic way in order to design an enantioselective actuator. Mandelic acid has been used in these first proof-of-principle experiments, but the approach can be extended to other chiral molecules, as long as their structures can be encoded in the metal. The mesoporous morphology of 
the metal layer ensures a significant difference in electroconversion of the two enantiomers, which is directly transduced into a different bending rate. The discrimination is optimal at the timescale of minutes. However, the kinetics of bending of the designed hybrid material strongly depends on the following three main factors: (i) the concentration of analyte; (ii) the difference of applied potential between the two feeder electrodes; (iii) the density of imprinted sites and the total electroactive surface area of the chiral imprinted mesoporous platinum. Therefore, playing with these three different parameters allows optimizing the bending kinetics for a given analyte. For long reaction times, both enantiomers will eventually result in the same degree of bending, because the actuator reacting with the correct enantiomer reaches its state of maximum bending. No significant difference of bending distance is observed at any time for non-imprinted Pt-PPy. This study describes a new concept where bipolar electrochemistry is used for enantioselective actuation based on a bilayer hybrid material. It can be considered as a mimic of certain biological processes where molecular recognition is combined with mechanical action and therefore opens up promising perspectives for the design of devices with sophisticated functionalities.

We thank the Vidyasirimedhi Institute of Science and Technology (VISTEC), FRC for SEM EDS mapping. This work was supported by the bilateral PICS program of CNRS and also by the Initiative of Excellence IdEx Bordeaux (ANR-10-IDEX-03-02). Chu. W. thanks the Thailand Research Fund (TRF) (MRG6180099) and TTSF research project supported by Thailand Toray Science Foundation. S.A. acknowledges a Ph.D. cotutelle scholarship from Campus France, the French Embassy in Thailand and VISTEC. This project has also been funded by the European Research Council (ERC) under the European Union's Horizon 2020 research and innovation program (grant agreement $n^{\circ}$ 741251, ERC Advanced grant ELECTRA).

Conflicts of interest

There are no conflicts to declare.

Notes and references

$\ddagger$ Sunpet Assavapanumat and Bhavana Gupta contributed equally.

1) (a) B. Han, Y. L. Zhang, L. Zhu, Y. Li, Z. C. Ma, Y. Q. Liu, X. L. Zhang, X. W. Cao, Q. D. Chen, C. W. Qiu, H. B. Sun, Adv. Mater. 2018, 31, 1806386. (b) M. S. Wang, G. Xu, G. C. Guo, Chem.Commun. 2010, 46, 361-376. (c) F. Hoffmann, M. Cornelius, J. Morell, M. Fröba, Angew. Chem. Int. Edit. 2006, 45. 3216-3251. (d) S. Inagaki, S. Guan, T. Ohsuna, O. Terasaki, Nature 2002, 416, 304-307. (e) H. Wang, Y. Liang, M. Gong, Y. Li, W. Chang, T. Mefford, J. Zhou, J. Wang, T. Regier. F. Wei, H. Dai, Nat. Commun. 2012, 3, 917. (f) J. Park, H. S. N. Jayawardena, X. Chen, K. W. Jayawardana, M. Sundhoro, E. Ada, M. W. Yan, Chem. Commun. 2015, 51, 2882-2885.

2) (a) M. Boyvat, J. S. Koh, R. J. Wood, Sci. Robot. 2017, 2, eaan1544. (b) N. Ruecha, J. Lee, H. Chae, H. Cheong, V. Soum, f P. Preechakasedkit, O. Chailapakul, G. Tanev, J. Madsen, N.

Rodthongkum, O. S. Kwon, K. Shin, Adv. Mater. Technol. 2017, 2, 1600267. (c) B. Gupta, B. Goudeau, A. Kuhn, Angew. Chem. Int. Edit. 2017, 56, 14183-14186. (d) X. Shen, L. Zhu, N. Wang, H. Tang, Chem. Commun. 2012, 48, 788-798. (e) M. Fuchiwaki, J. G. Martinez, T. F. Otero, Electrochim. Acta 2016, 195, 9-18.

3) G. A. Jacobson, K. C. Yee, D. Premilovac, S. Rattigan, Drug Test Anal. 2014, 6, 563-567. 
4) (a) M. Yoshikawa, K. Tharpa, Ş. O. Dima, Chem. Rev. 2016, 116, 11500-11528. (b) E. Yilmaz, K. Haupt, K. Mosbach, Angew. Chem. Int. Edit. 2000, 39, 2115-2118. (c) B. Sellergren, Angew. Chem. Int. Edit. 2000, 39, 1031-1037. (d) L. Chen, S. Xu, J. Li, Chem. Soc. Rev. 2011, 40, 2922-2942.

5) (a) T. Takagishi, I. M. Klotz, Biopolymers 1972, 11, 483-491. (b) R. Arshady, K. Mosbach, Makromol. Chem. 1981, 182, 687-692.

6) (a) P. Marchetti, M. F. J. Solomon, G. Szekely, A. G. Livingston, Chem. Rev. 2014, 114, 1073510806. (b) Y. Fuchs, O. Soppera, K. Haupt, Anal. Chim. Acta 2012, 717, 7-20. (c) J. Ashley, M. A. Shahbizi, K. Kant, V. A. Chidambara, A. Wolff, D. D. Bang, Y. Sun, Biosens. Bioelectron. 2017, 91, 606615.

7) (a) S. Arnaboldi, M. Magni, P. R. Mussini, Curr. Opin. Electrochem. 2018, 8, 60-72. (b) Y. Fang, C. Li, J. Bo, J. Henzie, Y. Yamauchi, T. Asahi, ChemElectroChem 2017, 4, 1832-1835. (c) C. Wattanakit, Curr. Opin. Electrochem. 2018, 7, 54-60. (d) M. Y. Mulla, E. Tuccori, M. Magliulo, G. Lattanzi, G. Palazzo, K. Persaud, L. Torsi, Nat. Commun. 2015, 6. 6010.(e) G. A. Attard, J Phy. Chem. B 2001, 105, 3158-3167. (f) J. A. Switzer, H. M. Kothari, P. Poizot, S. Nakanishi, E. W. Bohannan, Nature 2003, 425, 490-493.

8) S. C. Stinson, Chem. Eng. News 1992, 70, 46-79.

9) (a) G. Vasapollo, R. D. Sole, L. Mergola, M. R. Lazzoi, A. Scardino, S. Scorrano, G. Mele, Int. J. Mol . Sci. 2011, 12, 5908-5945. (b) M. Dabrowski, M. Cieplak, P. S. Sharma, P. Borowicz, K. Noworyta, W. Lisowski, F. D. Souza, A. Kuhn, W. Kutner. Biosens. Bioelectron. 2017, 94, 155-161.

10) (a) A. J. Gellman, ACS Nano 2010, 4, 5-10. (b) O. A. Hazzazi, G. A. Attard, P. B. Wells, J. Mol. Catal. A Chem. 2004, 216, 247-255. (c) E. W. Bohannan, H. M. Kothari, I. M. Nicic, J. A. Switzer, J. Am. Chem. Soc. 2004, 126, 488-489.

11) (a) H. Jiang, J. Ma, C. Li, Adv. Mater. 2012, 24, 4197-4202.(b) M. A. Jalebi, M. I. Dar, A. Sadhanala, S. P. Senanayak, F. Giordano, S. M. Zakeeruddin, M. Grätzel, R. H. Friend, J. Phys. Chem. Lett. 2016, 7, 3264-3269. (c) A. A. Melvin, K. Illath, T. Das, T. Raja, S. Bhattacharyya, C. S. Gopinath, Nanoscale 2015, 7, 13477-13488.

12) (a) C. Wattanakit, Y. B. S. Côme, V. Lapeyre, P. A. Bopp, M. Heim, S. Yadnum, S. Nokbin, C. Warakulwit, J. Limtrakul, A. Kuhn, Nat. Commun. 2014, 5, 3325. (b) T. Yuttalekha, C. Wattanakit, V. Lapeyre, S. Nokbin, C. Warakulwit, J. Limtrakul, A. Kuhn, Nat. Commun. 2016, 7, 12678. (c) C. Wattanakit, T. Yutthalekha, S. Assavapanumat, V. Lapeyre, A. Kuhn, Nat. Commun. 2017. 8, 2087. (d) S. Assavapanumat, T. Yutthalekha, P. Garrigue, B. Goudeau, V. Lapeyre, A. Perro, M. Sojic, C. Wattanakit, A. Kuhn, Angew. Chem. Int. Ed. 2019, 58, 3471-3475. (e) L. D. Pachón, I. Yosef, T. Z. Markus, R. Naaman, D. Avnir, G. Rothenberg, Nat. Chem. 2009, 1, 160-164. (f) D. Avnir, Adv. Mater. 2018, 30, 1706804.

13) J. Ding, D. Zhou, G. Spinks, G. Wallace, S. Forsyth, M. Forsyth, D. MacFariane, Chem. Mater. 2003. 15, 2392-2398.

14) (a) S. Inagi, Y. Ishiguro, M. Atobe, T. Fuchigami, Angew. Chem. Int. Ed. 2010. 49, 10136-

10139. (b) S. Chen, G. Wantz, L. Bouffier, J. Gao, ChemElectroChem 2016, 3, 392-398.

15) B. Gupta, B. Goudeau, P. Garrigue, A. Kuhn, Adv. Funct. Mater. 2018, 28, 1705825.

16) L. Zhang, B. Gupta, B. Goudeau, N. Mano, A. Kuhn, J. Am. Chem. Soc. 2018, 140, 1550115506. 
17) T. Yutthalekha, C. Warakulwit, J. Limtrakul, A. Kuhn, Electroanal. 2015, 27, 2209-2213.

18) T. Fuchigami, S. Inagi, M. Atobe, Fundamentals and Application of Organic Electrochemistry, John Willey\& Sons Ltd, West Sussex, 2015.

Table of Contents

A hybrid film composed of chiral encoded mesoporous platinum and polypyrrole shows differential wireless actuation as a function of the chiral nature of an enantiomer present in solution. 